\title{
OPEN Patterns of enhancement in paretic shoulder kinematics after stroke with musical cueing
}

\author{
Shinil Kang ${ }^{1}$, Joon-Ho Shin ${ }^{2}$, In Young Kim ${ }^{1}$, Jongshill Lee ${ }^{1}$, Ji-Yeoung Lee ${ }^{3}$ \& Eunju Jeong ${ }^{4,5} \bowtie$
}

Musical cueing has been widely utilised in post-stroke motor rehabilitation; however, the kinematic evidence on the effects of musical cueing is sparse. Further, the element-specific effects of musical cueing on upper-limb movements have rarely been investigated. This study aimed to kinematically quantify the effects of no auditory, rhythmic auditory, and melodic auditory cueing on shoulder abduction, holding, and adduction in patients who had experienced hemiparetic stroke. Kinematic data were obtained using inertial measurement units embedded in wearable bands. During the holding phase, melodic auditory cueing significantly increased the minimum Euler angle and decreased the range of motion compared with the other types of cueing. Further, the root mean square error in the angle measurements was significantly smaller and the duration of movement execution was significantly shorter during the holding phase when melodic auditory cueing was provided than when the other types of cueing were used. These findings indicated the important role of melodic auditory cueing for enhancing movement positioning, variability, and endurance. This study provides the first kinematic evidence on the effects of melodic auditory cueing on kinematic enhancement, thus suggesting the potential use of pitch-related elements in psychomotor rehabilitation.

Stroke is a leading cause of long-term functional disability ${ }^{1}$, resulting in increased dependency ${ }^{2}$ and social isolation $^{3,4}$ as well as decreased quality of life of patients ${ }^{5,6}$. Approximately $80 \%$ of stroke survivors have upperlimb dysfunction, varying from gross to complex and fine motor movements ${ }^{7}$. Post-stroke upper-limb rehabilitation remains challenging ${ }^{8-11}$, and the diverse approaches and methodologies have yielded controversial results $^{11,12}$. Recently, physicians and researchers have explored the potential of using diverse types of sensory cueing as a complement to conventional, vision-oriented approaches for motor rehabilitation ${ }^{13-15}$. Auditory stimulation has especially shown beneficial effects in enhancing movement execution ${ }^{16-20}$. Such stimuli may be perceived from all directions and reach across distances, making them perceptible even when the patient is not consciously or attentively listening ${ }^{21}$. Further, they immediately increase the excitability and readiness of motor execution, entrain the periodicity of movement patterns, and allow individuals to anticipate and prepare for the forthcoming movement ${ }^{22,23}$.

Auditory-motor enhancement is believed to have multifaceted mechanisms. At the perceptual level, auditory stimuli reach the brain faster than visual and tactile stimuli ${ }^{24-26}$. The auditory system has a high temporal resolution $^{27,28}$ and easily synchronises with the temporal periodicity of movements ${ }^{29,30}$. At the neuronal level, auditory cueing modulates neuromagnetic $\beta$ oscillations $s^{31,32}$, recruits movement-specific motor neurons s $^{33}$, and increases the variability in musculoskeletal activation pattern ${ }^{34}$. Most important, neural substrates involved in auditory processing closely communicate with those modulating motor timing, sequencing, and execution ${ }^{35-37}$. This is evidenced by the presence of a cortico-subcortical network that involves the putamen, supplementary motor area, premotor cortex, and auditory corte $\mathrm{x}^{38-41}$. Such auditory-motor connectivity is well represented and adopted in clinical studies. For example, rhythmic auditory stimulation (RAS), one of the techniques used in neurologic music therapy, leads to enhanced lower- and upper-extremity movements in patients with neurological impairments ${ }^{42-44}$. Specific to the upper limbs, movement timing ${ }^{45}$ and movement trajectory smoothness ${ }^{46}$ and velocity ${ }^{45,47}$ were greatly improved when RAS training was used in previous studies.

However, research on pitch-related elements has been relatively sparse. Perception of pitch and melody is part of the intrinsic nature of humans, is based on tonotopic representation in the auditory cortex ${ }^{48-50}$, and is

${ }^{1}$ Department of Biomedical Engineering, Hanyang University, Seoul, South Korea. ${ }^{2}$ National Rehabilitation Centre, Seoul, South Korea. ${ }^{3}$ Department of Medical Science, Graduate School, Soon Chun Hyang University, Asan, South Korea. ${ }^{4}$ Department of Music and Science for Clinical Practice, Hanyang University, Seoul, South Korea. ${ }^{5}$ College of Interdisciplinary Industrial Studies, Hanyang University, Seoul, South Korea. ${ }^{\varpi}$ email: ejeong@hanyang.ac.kr 


\begin{tabular}{|c|c|c|c|c|c|c|c|c|c|}
\hline \multirow[b]{2}{*}{ Kinematic parameter } & \multicolumn{3}{|c|}{ Abduction } & \multicolumn{3}{|c|}{ Holding } & \multicolumn{3}{|c|}{ Adduction } \\
\hline & NAC & RAC & MAC & NAC & RAC & MAC & NAC & RAC & MAC \\
\hline \multicolumn{10}{|l|}{ ROM } \\
\hline M & 127.27 & 128.61 & 128.33 & 13.01 & 10.25 & 8.32 & 119.88 & 121.55 & 123.57 \\
\hline SD & 53.58 & 52.33 & 53.35 & 11.84 & 10.42 & 8.32 & 56.91 & 54.82 & 55.91 \\
\hline \multicolumn{10}{|l|}{ MIN } \\
\hline M & 4.63 & 5.15 & 5.33 & 119.43 & 122.95 & 125.48 & 4.65 & 5.01 & 5.00 \\
\hline SD & 10.53 & 12.41 & 11.12 & 57.00 & 54.63 & 55.98 & 9.89 & 12.52 & 10.85 \\
\hline \multicolumn{10}{|l|}{ MAX } \\
\hline M & 131.91 & 133.79 & 133.68 & 135.63 & 135.78 & 135.50 & 124.60 & 126.67 & 128.60 \\
\hline SD & 54.48 & 52.29 & 53.79 & 54.74 & 52.10 & 54.22 & 57.87 & 54.38 & 56.14 \\
\hline \multicolumn{10}{|l|}{ RMSE } \\
\hline M & 11.91 & 12.97 & 12.78 & 1.87 & 1.62 & 1.24 & 13.37 & 13.08 & 13.69 \\
\hline SD & 8.63 & 8.67 & 8.21 & 1.64 & 1.36 & 1.18 & 10.96 & 9.60 & 9.91 \\
\hline \multicolumn{10}{|l|}{ Duration } \\
\hline $\mathrm{M}$ & 295.44 & 300.36 & 312.59 & 259.60 & 232.90 & 199.63 & 330.24 & 351.84 & 373.36 \\
\hline SD & 66.57 & 64.22 & 64.38 & 90.02 & 82.63 & 55.01 & 73.95 & 90.27 & 93.52 \\
\hline
\end{tabular}

Table 1. Descriptive statistics of kinematic parameters. ROM range of motion, $M I N$ minimum Euler angle, $M A X$ maximum Euler angle, $R M S E$ root mean square error, $N A C$ no auditory cueing, $R A C$ rhythmic auditory cueing, $M A C$ melodic auditory cueing, $M$ mean, $S D$ standard deviation.

associated with space perception ${ }^{51,52}$. Thus, pitch and melody can provide a cognitive representation of movement in terms of spatial location and direction ${ }^{53}$. For example, ascending and descending melodic contours cue upward and downward movements. The effects of pitch-related elements have also been demonstrated in clinical studies. Patterned sensory enhancement utilises rhythmic, melodic, and harmonic elements to provide temporal, spatial, and dynamic information about the movement ${ }^{54,55}$ and has been applied to upper-limb rehabilitation ${ }^{55-61}$. However, the results have been inconsistent, which might be due to the non-specific use of musical elements. Moreover, to the best of our knowledge, element-specific effects have not been examined using a controlled experimental design to date.

Additionally, most previous studies employed conventional observations, rating scales, or questionnaires for evaluation ${ }^{56-60}$, which are inherently subjective and vary depending on the evaluator(s) ${ }^{62}$. These methodologies hardly allow the measurement of kinematic changes throughout upper-limb movements during auditory cueing; thus, more sophisticated methodologies are needed. In the present study, we employed inertial measurement units (IMUs) to quantify the kinematics of repetitive shoulder abduction, holding, and adduction movements in hemiparetic stroke patients. The purpose of the present study was to examine the effects of (1) no auditory cueing (NAC), (2) rhythmic auditory cueing (RAC), and (3) melodic auditory cueing (MAC) on the kinematic parameters of shoulder movement, including range of motion (ROM), minimum Euler angle (MIN), maximum Euler angle (MAX), duration, and root mean square error (RMSE).

\section{Results}

Table 1 shows the descriptive findings. During the holding phase, there existed a significant main effect of the cue on the $\operatorname{ROM}\left[F_{(2,30)}=8.801, p<0.01\right]$ and $\operatorname{MIN}\left[F_{(2,30)}=9.087, p<0.01\right]$ (Fig. 1). A pairwise post-hoc comparison with Bonferroni correction revealed that the ROM was greater in NAC than in MAC $(p<0.0167)$, whereas the MIN was higher in MAC than in NAC $(p<0.0167)$. A higher MIN indicated that MAC can assist in the maintenance of shoulder holding at a higher position. The smaller ROM observed in MAC than in NAC and RAC indicated that MAC helped maintain a less variable shoulder angle with less Euler angle drift.

Further, there existed a significant main effect of cue on the duration $\left[F_{(2,30)}=22.028, p<0.001\right]$. A pairwise comparison showed that during the holding phase, the duration of shoulder movement was significantly longer in NAC than in RAC $(p<0.0167)$, in NAC than in MAC $(p<0.0167)$, and in RAC than in MAC $(p<0.0167)$. Interestingly, there also existed a significant main effect of cue on the duration $\left[F_{(2,30)}=6.724, p<0.01\right]$ in the adduction phase. Although the pairwise comparison yielded insignificant results for all pairs $(p>0.0167)$, the duration associated with MAC was the longest, followed by that associated with RAC and then NAC.

We also performed a movement variability (MV) analysis. General approaches to MV quantification in upper-limb movements are typically based on the distribution of angle, acceleration, and velocity ${ }^{63}$. During the holding phase, there was a significant main effect of cueing on the $\operatorname{RMSE}\left[F_{(2,30)}=8.109, p<0.01\right]$. A pairwise post-hoc comparison revealed significantly smaller RMSE values in MAC than in NAC $(p<0.0167)$ and RAC $(p<0.0167)$ (Fig. 1).

We calculated RMSE as a MV indicator because Euler angle values are sensitive for the measurement of movement acceleration and deceleration. Figure 2 shows the Euler angle variability between the affected and intact sides (black line) during the holding phase, as compared with the fitted curve (red line). The Euler angle of the affected side showed a sudden decrease after the arm approached the highest position and before the start 
A

ROM

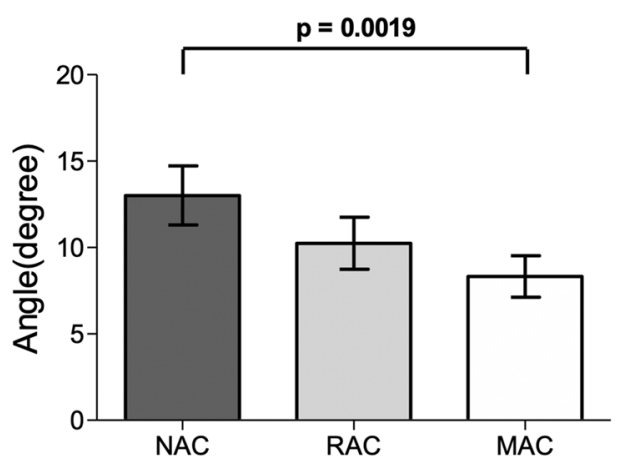

C

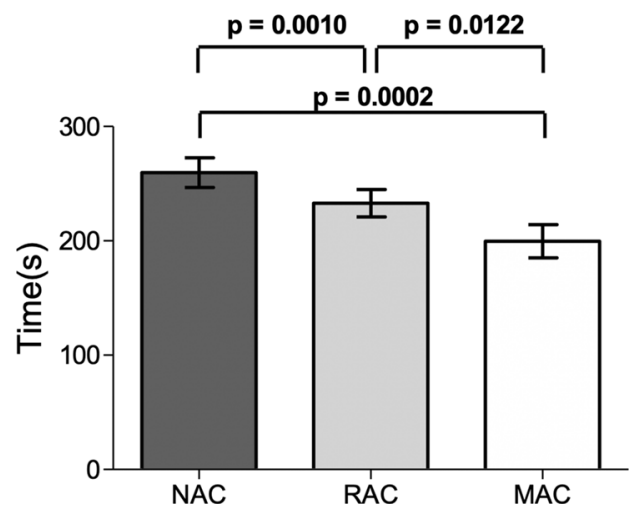

B

MIN

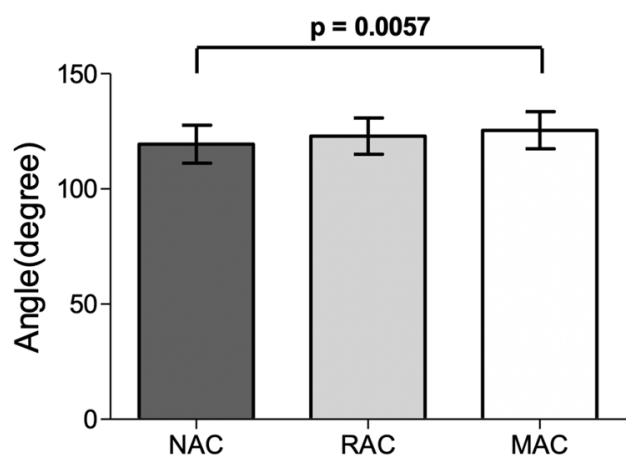

RMSE

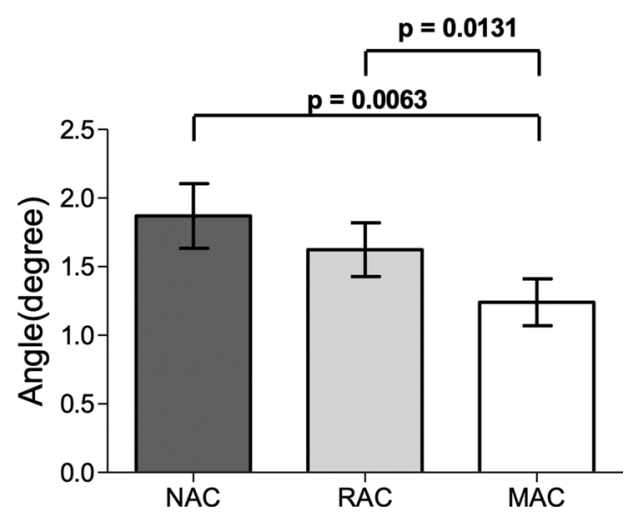

Figure 1. Significant differences of kinematic parameters of movement. The graphs show means with standard errors. (A) ROM during the holding phase. (B) MIN during the holding phase. (C) Duration during the holding phase. (D) RMSE during the holding phase. $P$ values are provided for significant differences $(p<0.0167)$.
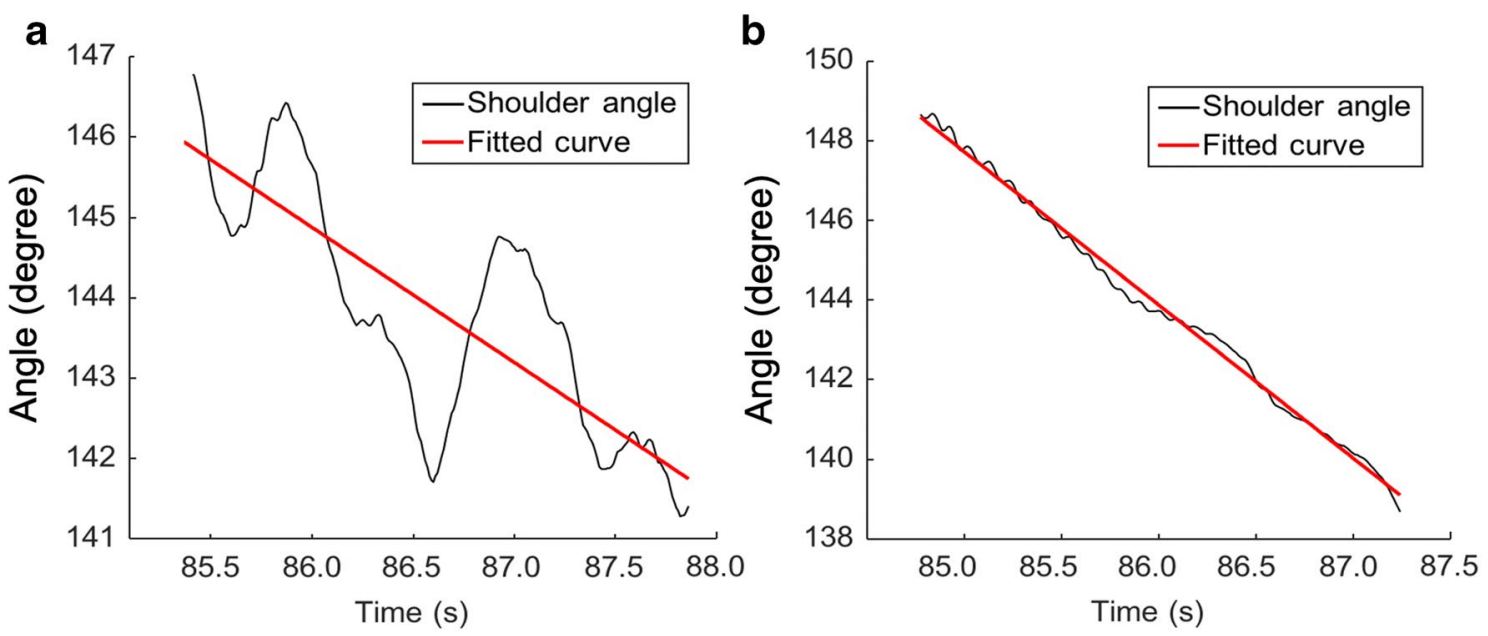

Figure 2. Comparison of Euler angle data of shoulder movement. (A) Data obtained from severely affected patients' shoulders. (B) Data obtained from moderately affected patients' shoulders.

of the adduction phase. This finding was similar to observations made in previous studies that investigated the stroke-specific kinematic features of shoulder movements ${ }^{63-66}$.

Further, we scrutinised the Euler angle profiles during the holding phase. Figure 3 shows representative Euler angle magnitude profiles. A visual inspection revealed that, in all patients except P2 and P11, less Euler angle profile variability was observed in MAC than in NAC. Moreover, in most patients except P2, P9, P10, P11, and P16, less Euler angle profile variability was observed in MAC than in RAC. 




Figure 3. Movement variability profile using Euler angle data. Representative RMSE profiles of the magnitudes of the Euler angle of 16 patients during the shoulder movement holding phase are shown. The $\mathrm{x}$-axis displays $100 \%$ of the movement cycle: $0 \%$ and $100 \%$ are the shoulder movement holding times. The $y$-axis displays the normalised Euler angle values. Normalised Euler angle values were used to enhance the comparison among patients. The left column shows the NAC condition; the middle column shows the RAC condition; and the right column shows the MAC condition. 


\section{Discussion}

In the present study, we used IMUs to measure the complex kinematic characteristics of shoulder abduction, holding, and adduction movements occurring in conjunction with NAC, RAC, and MAC. The findings revealed that MAC significantly increased the MIN and decreased the ROM and RMSE during the holding phase. MAC also decreased the duration of the holding phase. Taken together, these findings indicated that MAC enhances movement positioning, decreases movement variability, and increases movement anticipation and preparation.

First, the results during the holding phase showed that the MIN was significantly higher in MAC than in NAC and that the ROM was significantly greater in NAC than in MAC. These findings collectively indicated that the MIN of the shoulder was maintained at the highest position with less movement deviation in MAC. The enhancement observed in MAC occurred because the participants were provided with information about movement directions until they reached their maximum shoulder angle. These results are similar to those of previous studies that examined the effects of music cognition on movement, demonstrating that pitch contours can increase the cognitive awareness of spatial information during motion ${ }^{51}$ and actually enhance limb positioning during the execution of vertical movements ${ }^{67}$.

Second, the RMSE was significantly smaller in MAC than in NAC and RAC during the holding phase. These findings indicated that the observed Euler angle data, which can sensitively detect movement acceleration and deceleration, were best fitted to the predicted linear regression model in MAC; thus, MAC yielded the smallest RMSE. Note that during the holding phase, MAC involved an isochronous rhythm combined with a $740-\mathrm{Hz}$ stationary contour, which is higher than the tone used in RAC $(440 \mathrm{~Hz}$, see Fig. 1). Thus, the closer RMSE values of the observed data to the predicted model in MAC was possibly due to the effect of high-pitched sequences embedded in the middle of a melodic contour on the cognitive representation of movement in terms of spatial location ${ }^{53}$, which allowed the participants to sustain their shoulders with less movement variability. In line with the current findings, a previous study reported that a melody cue was best for unconstrained point-to-point hand movements, prompting less variable hand movements from the initial to the final position within a given time period $^{68}$. Moreover, previous studies have demonstrated that auditory cueing significantly decreases movement variability ${ }^{45,46,69-72}$. For example, Thaut et al. reported that the kinematic data obtained in auditory cueing fit the predicted model significantly better than those from a no auditory cueing condition. This result was possibly due to the entrainment of movement execution patterns with the patterns embedded in the rhythm ${ }^{46}$.

Third, the duration was significantly shorter in MAC than in NAC, in RAC than in NAC, and in MAC than in RAC. RAC consists of an isochronous rhythm, whereas MAC consists of pitch contours presented with an isochronous rhythm. In the current study, this rhythm pattern seemed to play a role in movement anticipation and preparation in both RAC and MAC. In a review study, Avanzino et al. ${ }^{73}$ reported that rhythm provides a temporal structure that enhances motor timing, motor sequencing, and movement control. Moreover, isochronous rhythm was reported to induce a temporal locking or entrainment process between motor movements and the external auditory rhythm ${ }^{22}$.

In addition, the shorter duration of RAC and MAC can be interpreted as a cognitive influence of musical cueing on anticipation and preparation for movement. Schaffert et al. ${ }^{74}$ proposed that auditory rhythm can prime the motor system and facilitate further anticipation of and preparation for cyclic movements ${ }^{75}$. Such influences, when observed in healthy and clinical (e.g. post-stroke) populations, manifest through the activation or establishment of alternative pathways ${ }^{76-78}$. The available findings implied that cognitive involvement in auditory processing (i.e. anticipation) plays a role in motor preparation and execution ${ }^{79,80}$. Importantly, as the pitch contours embedded in RAC are more salient to providing the structure of movement, patients are more likely to anticipate and prepare for the forthcoming movement. In general, music involves a high-order temporal organisation of sound events, of which allows individuals to make predictions about future events and raises expectations ${ }^{37}$. Previous studies that employed melodic feedback in post-stroke arm rehabilitation also reported improved smoothness of movement because of such anticipation ${ }^{81,82}$.

Collectively, MAC led to an effective, stable maintenance of the shoulder in the desired position during the holding phase. The closer fit observed in MAC indicated a smaller movement variability possibly due to the effect of pitch in addition to the effect of rhythm. The shorter movement time in MAC indicates that rhythm components play a role in movement anticipation and preparation and are more prominent when presented with melodic components. Compared with MAC, RAC showed significant enhancement only in movement time.

Our study had some limitations. We performed parameter extraction for the Euler angles of the sagittal axis because this axis provides more relevant information about our target movement. Obtaining similar data from two other axes and involving another body part (wrist, hand, neck, etc.) may provide a more comprehensive index for the movement features of the affected shoulder (e.g. post-stroke compensatory strategies). However, this study was a preliminary attempt to quantify the kinematic parameters of repetitive paretic shoulder abduction, holding, and adduction in post-stroke patients. As the effects of musical cueing on the kinematic changes of upper-limb movements have not yet been specified and the scope of their application has been limited, the present study provides important information about the specific roles of the elements as sensory cues and promotes a more appropriate use of the elements for upper-limb rehabilitation. Future studies are needed to find a more comprehensive index of the relationship between musical cueing and functional upper-limb movements and to develop a more efficient and targeted upper-limb patterned sensory enhancement protocol. Lastly, combining musical cueing with visually oriented virtual reality rehabilitation may yield better psychomotor rehabilitation.

In conclusion, auditory cueing led to enhanced movements, across all parameters, when compared with movements performed without auditory cueing in this study. In particular, MAC effectively increased the MIN but decreased the RMSE and duration in the holding phase. These findings seem to be associated with increased movement positioning, decreased movement variability, and enhanced movement anticipation and preparation. Such findings suggest the potential of pitch-related components for directing specific movement parameters and 
A

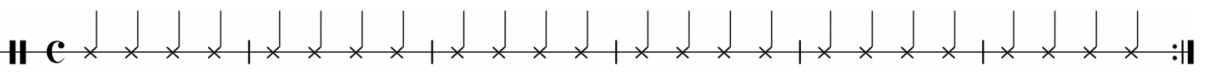

B

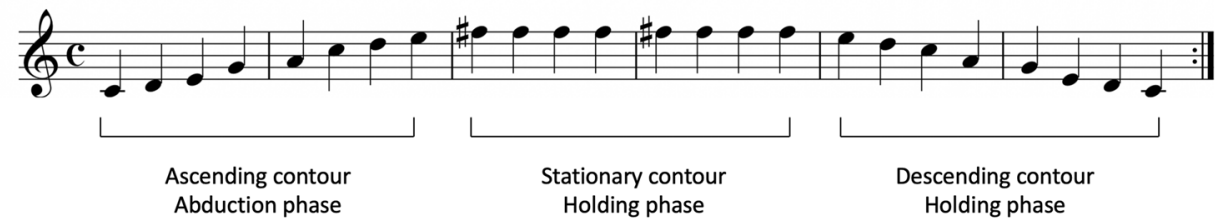

Figure 4. An example of auditory cueing. (A) Rhythmic auditory cueing. (B) Melodic auditory cueing.

the potential use of musical cueing in psychomotor rehabilitation. Given that muscle endurance is necessary to allow smooth control of movements, which is typically impaired in stroke patients (as evidenced by their daily functional movements ${ }^{83-85}$, the current findings can be used for the conclusive measurement of motor function recovery and for rehabilitation training ${ }^{86}$.

\section{Methods}

Participants. This study was approved by the Institutional Review Board at the National Rehabilitation Centre and performed in accordance with relevant guidelines and regulations. All participants provided written informed consent, in accordance with the Declaration of Helsinki. Informed consent to publish identifying images was also obtained from participants. Eighteen patients with hemiparetic stroke $(\mathrm{Male}=10$, Female $=8)$ volunteered to participate in the study and were recruited from the rehabilitation centre. The patients were eligible if they had upper-limb hemiparesis secondary to first-ever ischaemic or haemorrhagic stroke, Brunnstrom Stages of Motor Recovery scores $>2$ for the affected proximal upper limb, the cognitive ability to understand and follow instructions, and the capability and willingness to participate in the experiment. Patients who had more than 3 months of regular involvement in musical activities and/or professional training and those with sensory impairments were excluded from the study. Two of the 18 patients were excluded because of unreliable signalto-noise ratios. The average age of the patients was $49.78(\mathrm{SD}=15.55)$ years, and the average time since stroke was $15.22(\mathrm{SD}=12.82)$ months. The patients had an average of $12.28(\mathrm{SD}=2.80)$ years of education, an average Brunnstrom Stage of Motor Recovery score of $4.67(\mathrm{SD}=0.94)$, and an average shoulder abduction ROM of $138.33^{\circ}(\mathrm{SD}=29.06)$.

Movement task and auditory stimuli. To examine the immediate effects of auditory cueing on paretic shoulder movements, we selected shoulder abduction, holding, and adduction movements as previous studies had reported the stroke-specific kinematic features of shoulder movements ${ }^{87-90}$. A visual avatar, from a thirdperson perspective, was provided to guide the movement. The avatar was programmed to raise its arms from the side to $180^{\circ}$ (abduction phase, $3 \mathrm{~s}$ ), maintain its arms at that position (holding phase, $3 \mathrm{~s}$ ), and then slowly lower its arms back to the side (adduction phase, $3 \mathrm{~s}$ ). The participants were instructed to perform the movements in conjunction with the avatar seen on the computer screen. The avatar was generated using a model of Rehab Master (Rehab Master, Seoul, Korea) and programmed using Unity (Unity Technologies, Copenhagen, Denmark).

Two types of auditory stimuli were used as auditory cues and presented via two stereo speakers. RAC consisted of a series of 50-ms tones with an inter-stimulus interval (ISI) of $200 \mathrm{~ms}$ (i.e. a beep sound on digital metronome at an audio frequency of $440 \mathrm{~Hz}$ ). MAC consisted of a series of tones presented using the same isochronous time pattern as the RAC but at different frequencies. In the present study, RAC and MAC have definite pitches and the same isochronous time pattern, so they might also be labelled monotonic rhythm and melodic rhythm, respectively. However, we used the terms rhythmic and melodic in accordance with the terminologies used in the existing literature. RAC or RAS is a frequently used psychomotor rehabilitation technique. The rhythmic stimuli employed in these studies are mainly analogue or digital metronome beats ${ }^{69,76,91}$ comprising tones presented at the same pitch and with an isochronous time pattern. In addition, considering the general definition of melody as a combination of pitch and rhythm, it might be better to use the term MAC.

MAC consisted of ascending, stationary, and descending contours generated to musically demonstrate the angle of shoulder abduction, holding, and adduction, respectively. In typical participants, shoulder abduction involves three different types of muscle-the supraspinatus, deltoid, and trapezius and serratus anterior. The supraspinatus initiates from $0^{\circ}$ to $15^{\circ}$, the deltoid functions from $15^{\circ}$ to $90^{\circ}$, and the trapezius and serratus anterior are activated from $90^{\circ}$ to $180^{\circ}$. In addition, the glenohumeral joint contributes $90^{\circ}-120^{\circ}$ of shoulder abduction ${ }^{93,94}$. The range of shoulder movement $\left(0^{\circ}-180^{\circ}\right)$ was divided into ten equal degrees, and a 12-tone scale ( $\mathrm{C} 4$ to $\mathrm{F} \# 5)$ was assigned to each degree. As $0^{\circ}, 15^{\circ}, 90^{\circ}, 120^{\circ}$, and $180^{\circ}$ are important to indicate the muscle involvement in shoulder abduction, we selected the pitches that correspond to the degrees $(\mathrm{C} 4, \mathrm{D} 4, \mathrm{~A} 4, \mathrm{C} 5$, and F5\#). As $15^{\circ}$ corresponds to the microtone between C4\# and D4, we selected D. Additional tones were used to musically describe the spatial position and trajectory of shoulder movement, yielding an eight-tone ascending contour. The first four tones of the ascending contour (C4, D4, E4, and G4) were assigned to shoulder abduction from $0^{\circ}$ to $89^{\circ}$, and the second four tones (A4, C5, D5, and E5) were assigned to shoulder abduction from $90^{\circ}$ to $179^{\circ}$. The next eight tones of the stationary contour reflect the shoulder holding movement. As maximum effort is needed to hold the shoulder at the highest position (typically around $170^{\circ}-180^{\circ}$ ), F5\# was used to reflect a such effort. Figure 4 shows an example of RAC and MAC. The auditory stimuli were generated using a musical 

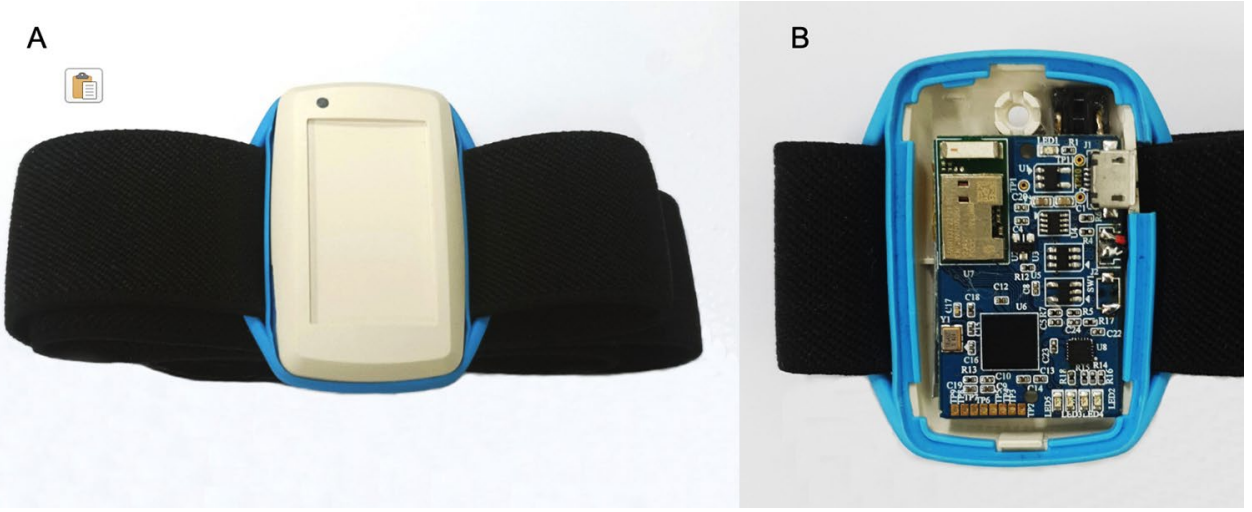

Figure 5. In-house-built H-IMU. (A) Cover closed. (B) Cover opened.
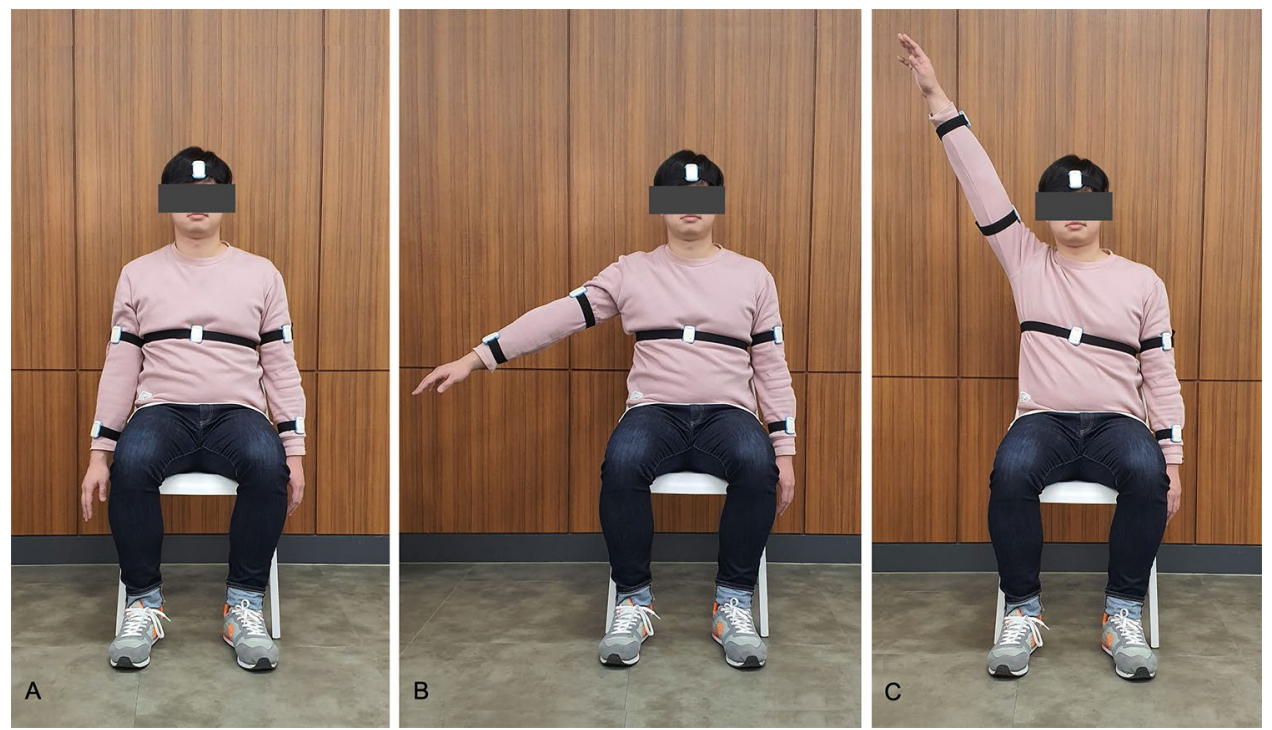

Figure 6. An individual demonstrating shoulder abduction with the H-IMUs attached. (A) Arm at the side. (B) Raising one arm halfway. (C) Reaching the arm towards the head.

instrument digital interface synthesizer connected to a Logic Pro X (Apple, Cupertino, CA, USA) and programmed in accordance with the avatar movements using Unity (Unity Technologies, Copenhagen, Denmark).

IMUs and equipment setup. To measure and analyse the effects of cueing on the participants' shoulder movements, we used spatiotemporal kinematic parameters. These parameters were provided by Hanyang IMU (H-IMU), which consists of an integrated 9-axis motion tracking sensor (MPU9250; InvenSense, San Jose, CA, USA), a microcontroller (MSP430F5338; Texas Instruments, Dallas, TX, USA), a Bluetooth module (PAN1321i; Panasonic, Osaka, Japan), and a lithium-ion battery (240 mAh, 3.7 V; DTP, Shenzhen, China). The device allows long-term data measurements (Fig. 5). A total of $6 \mathrm{H}$-IMUs were attached to the head, torso, arms, and forearms of each patient using Velcro straps and were positioned on the dorsal side of each arm and on the anterior portion of the head and torso. Signals associated with body movements were generated at $<20 \mathrm{~Hz}$, such that a sampling frequency of $100 \mathrm{~Hz}$ was sufficient to detect all movements. Thus, all H-IMU signals were measured using a sampling rate of $100 \mathrm{~Hz}$. The calculated Euler angles for each of the $6 \mathrm{H}$-IMUs were transmitted to a personal computer using Bluetooth communication. Figure 6 shows the H-IMUs and how they were attached to a participant.

In each experiment, the participants underwent a practice period (P1) and an experimental period (P2). P1 comprised three shoulder abduction-holding-adduction trials, in which the three types of cueing were presented. The P1 period allowed the participants to become familiar with the movements and the different types of cueing. P2 comprised three blocks of three cueing conditions. Five trials, each consisting of abduction, holding, and adduction phases, were blocked with one of the three cueing conditions and randomly presented to the participant while avoiding the consecutive presentation of the same cueing condition. The blocks requiring the movement of the affected and unaffected shoulders were presented in an alternating manner. 


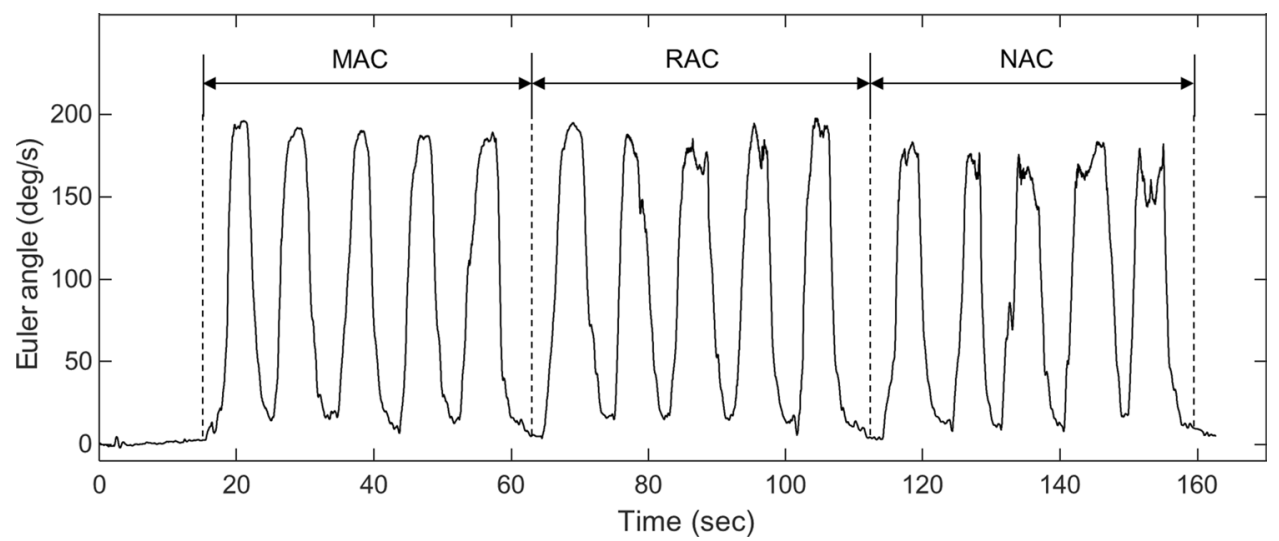

Figure 7. Kinematic recording of hemiparetic shoulder abduction, holding, and adduction movements across three types of cueing.

Euler angle calculation. Micro-electromechanical system inertial sensors have a direct-current bias and gain error because of the direct-current noise caused by powering of the electric circuit and environmental noise. To minimise the error associated with these noises, we calibrated the sensors using an in-house-manufactured calibration jig with 9 degrees of freedom $(\mathrm{DoFs})^{95}$. The calibrated data, c, can be obtained by multiplying the uncalibrated data, $\mathrm{u}$, including the original error by the compensated gain value, $\mathrm{K}$, through calibration and subtracting the offset error, b. These variables are defined in Eqs. (1-3), respectively. Finally, the calculated calibration equation is shown in Eq. (4)

$$
\begin{gathered}
u=\left[\begin{array}{lll}
u_{x} & u_{y} & u_{z}
\end{array}\right]^{T} \\
\mathrm{~K}=\left[\begin{array}{ccc}
k_{x} & 0 & 0 \\
0 & k_{y} & 0 \\
0 & 0 & k_{z}
\end{array}\right] \\
\mathrm{b}=\left[\begin{array}{lll}
b_{x} & b_{y} & b_{z}
\end{array}\right]^{T} \\
\mathrm{c}=\mathrm{Ku}-\mathrm{b}
\end{gathered}
$$

Thereafter, the Euler angles were calculated using a gradient descent algorithm to provide the quaternions based on tri-axis gyroscope, accelerometer, and magnetometer measurements, as shown in Eq. (5). Euler angles adopt the ' $Z Y X$ ' rotation sequence to convert quaternion frame rotations in radians ${ }^{96,97}$. Figure 7 shows an example of the calculated Euler angles associated with the three types of cueing

$$
\begin{aligned}
& \emptyset=\operatorname{atan} 2\left(2 \mathrm{q}_{3} \mathrm{q}_{4}-\mathrm{q}_{1} \mathrm{q}_{2}, 2 \mathrm{q}_{1}^{2}+2 \mathrm{q}_{4}^{2}-1\right) \\
& \theta=-\sin ^{-1}\left(2 \mathrm{q}_{2} \mathrm{q}_{4}+2 \mathrm{q}_{1} \mathrm{q}_{3}\right) \\
& \varphi=\operatorname{atan} 2\left(2 \mathrm{q}_{2} \mathrm{q}_{3}-2 \mathrm{q}_{1} \mathrm{q}_{4}, 2 \mathrm{q}_{1}^{2}+2 \mathrm{q}_{2}^{2}-1\right)
\end{aligned}
$$

where $\mathrm{q}_{\mathrm{x}}(\mathrm{x}=1,2,3,4)$ is the estimated orientation of the sensor frame relative to the earth frame.

Movement analysis. We extracted the kinematic parameters of movement during the three phases (abduction, holding, and adduction) of shoulder movement. Parameter extraction was performed on the Euler angles obtained from the frontal plane around the sagittal axis, which is the axis that provides more relevant information about the abduction, holding, and adduction movements of the shoulder. Figure 8 shows the points that were included in the movement analysis. The start point (SP) and end point (EP) indicate the initiation of the abduction phase and the cessation of the adduction phase, respectively, based on the minimum Euler angle values. Fiducial points (FP1, FP2) were defined to correspond with the initiation and cessation of the holding phase; FP1 represents the maximum point on the Euler angle graph for one cycle.

We selected FP2 values that satisfy the equations below, in which $f(x)$ represents the angle, $x$ represents the time point, and i represents the peak points between FP1 and EP. FP2 was not easy to define because the signalto-noise ratios were extreme in all participants. Based on our preliminary experimentation and simulations, we set a threshold $(\alpha)$ for the between-peak slope $(-0.03)$ and applied this to all data sets. Small peak points, with peak values below -0.03 , were not considered to be reliable FP2 values $(6,7)$ 


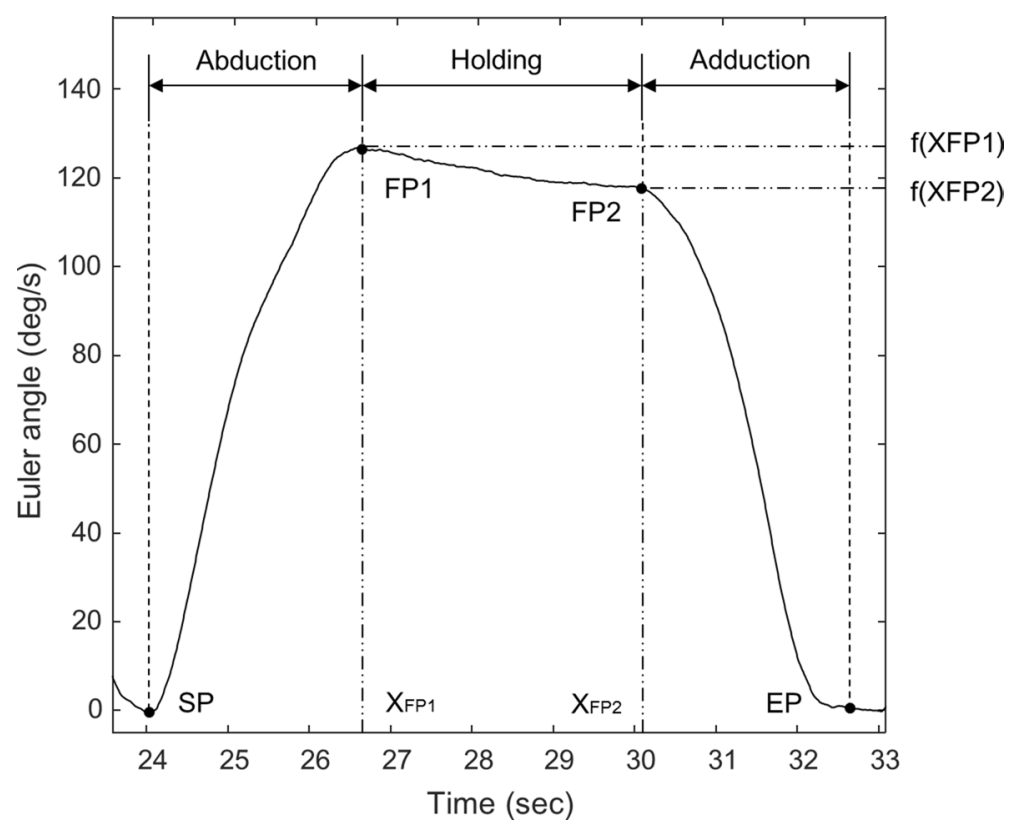

Figure 8. Sample raw Euler angle data obtained from one trial of shoulder abduction, holding, and adduction. $S P$ start point, $E P$ end point, $F P$ fiducial point.

\begin{tabular}{|l|l|l|}
\hline Kinematic parameter & Formula & Description \\
\hline ROM & $\max \left(\varnothing_{\text {phase }}\right)-\min \left(\varnothing_{\text {phase }}\right)$ & \\
\hline MIN & $\min \left(\varnothing_{\text {phase }}\right)$ & \\
\hline MAX & $\max \left(\varnothing_{\text {phase }}\right)$ & \\
\hline RMSE & $\sqrt{\frac{1}{n} \sum_{i=F P 1}^{F P 2}\left(\varnothing_{i}-\widehat{\varnothing}_{i}\right)^{2}}$ & Calculated using a linear regression model \\
\hline Duration & $t_{\text {endphase }}-t_{\text {startphase }}$ & Time spent to complete one phase of each trial \\
\hline
\end{tabular}

Table 2. Spatiotemporal features. $\varnothing_{\text {phase }}$ Euler angle values obtained during one phase of each trial, $n$ number of samples for the phase, $\widehat{\varnothing}$ linear regression value, $t$ time point, MIN minimum Euler angle of the phase, MAX maximum Euler angle of the phase, RMSE root mean square error.

$$
\begin{gathered}
\mathrm{P}=\left\{x_{i} \mid \frac{f\left(x_{F P 1}\right)-f\left(x_{i}\right)}{x_{F P 1}-x_{i}}<\alpha, \quad \mathrm{FP} 1 \leq \mathrm{i} \leq \mathrm{EP}\right\} \\
x_{F P 2}=\max (P), y_{F P 2}=f\left(x_{F P 2}\right), F P 2=\left(x_{F P 2}, y_{F P 2}\right)
\end{gathered}
$$

Based on previous studies in clinical populations that revealed the meaning of Euler angle values relative to motor movements ${ }^{98-100}$, we calculated the kinematic parameters using Euler angles (Table 2).

ROM. Shoulder movement is characterised by many DoFs and a wide ROM at the joints ${ }^{101}$. The ROM is important for assessing joint range, control, strength, and willingness to perform a movement ${ }^{102}$. The clinical implication of the kinematic ROM has recently been expanded to the determination of diagnoses and the success of therapeutic interventions. Also, previous studies have reported the validity and reliability of IMUs for measuring parameters of shoulder joint motion, such as the $\mathrm{ROM}^{103-105}$. In the present study, the ROM refers to the difference between the MIN and MAX, using the anatomical DoFs for human limbs ${ }^{64,106}$, and was calculated for each of the three movement phases. In the abduction and adduction phases, the ROM reflects the largest Euler angle, as arm raising was initiated from approximately $0^{\circ}$. During the holding phase, the ROM reflects the variance among the Euler angles.

Movement variability $(M V)$. General approaches to MV quantification in upper-limb movements are typically based on the distribution of characteristic values, such as angle, acceleration, and velocity ${ }^{63}$. Movement variability is associated with the variety of coordination patterns used to complete tasks ${ }^{65,66}$. For the current study, we calculated RMSEs using a linear regression model and considered the parameter as an indicator of MV. The reason that we considered RMSE as a measure of MV was that the Euler angle values measure movement acceleration and deceleration. Thus, the RMSE represents the difference between the generated linear regression 
model (fitted curve) and the obtained Euler angle data, which indicate the ideal movement trajectory and the raw data associated with shoulder movement, respectively. In the current study, MV during the abduction and adduction phases might indicate movement smoothness, while MV during the holding phase might indicate movement endurance.

Movement time (MT). MT refers to the time required to execute a movement and is defined as the interval between movement onset and movement offset, representing temporal efficiency ${ }^{107-109}$. In the present study, MT included the duration of shoulder abduction (time between the onset of arm raising and attaining the maximum shoulder angle, SP to FP1), shoulder holding (time of maintaining the maximum shoulder angle, FP1 to FP2), and shoulder adduction (time between the onset of arm lowering and back to the initial position, FP2 to EP; see Fig. 8 for details). In general, MT is associated with muscle endurance, which is the ability of a muscle to sustain repetitive isometric or isotonic contractions ${ }^{110}$.

Statistical analysis. The kinematic parameters from the affected side were used and categorised into the three phases of shoulder movement (abduction, holding, and adduction). A one-way repeated measures analysis of variance was performed to compare the kinematic parameters across the three cueing conditions (NAC, RAC, and MAC). All analyses were conducted using R (R Project for Statistical Computing, Vienna, Austria).

\section{Data availability}

The datasets generated and/or analysed during the current study are available from the corresponding author upon reasonable request.

Received: 8 February 2020; Accepted: 5 October 2020

Published online: 22 October 2020

\section{References}

1. Hong, K.-S. et al. Stroke Statistics in Korea: Part I. Epidemiology and risk factors: A report from the Korean Stroke Society and Clinical Research Center for Stroke. J. Stroke 15, 2-20 (2013).

2. Pollock, A. et al. Interventions for improving upper limb function after stroke. Cochrane Database Syst. Rev. 2014, CD010820 (2014).

3. Haun, J., Rittman, M. \& Sberna, M. The continuum of connectedness and social isolation during post stroke recovery. J. Aging Stud. 22, 54-64 (2008)

4. White, J. H., Attia, J., Sturm, J., Carter, G. \& Magin, P. Predictors of depression and anxiety in community dwelling stroke survivors: A cohort study. Disabil. Rehabil. 36, 1975-1982 (2014).

5. King, J. M. \& Hux, K. Attention allocation in adults with and without aphasia: Performance on linguistic and nonlinguistic tasks. J. Med. Speech. Lang. Pathol. 4, 245-256 (1996).

6. Franceschini, M., La Porta, F., Agosti, M. \& Massucci, M. Is health-related-quality of life of stroke patients influenced by neurological impairments at one year after stroke?. Eur. J. Phys. Rehabil. Med. 46, 389-399 (2010).

7. Handley, A., Medcalf, P., Hellier, K. \& Dutta, D. Movement disorders after stroke. Age Ageing 38, 260-266 (2009).

8. Trumbower, R. D., Ravichandran, V. J., Krutky, M. A. \& Perreault, E. J. Contributions of altered stretch reflex coordination to arm impairments following stroke. J. Neurophysiol. 104, 3612-3624 (2010).

9. Messier, S., Bourbonnais, D., Desrosiers, J. \& Roy, Y. Kinematic analysis of upper limbs and trunk movement during bilateral movement after stroke. Arch. Phys. Med. Rehabil. 87, 1463-1470 (2006).

10. Van Dokkum, L. et al. The contribution of kinematics in the assessment of upper limb motor recovery early after stroke. Neurorehabil. Neural Repair 28, 4-12 (2014).

11. Pulman, J., Buckley, E. \& Clark-Carter, D. A meta-analysis evaluating the effectiveness of two different upper limb hemiparesis interventions on improving health-related quality of life following stroke. Top. Stroke Rehabil. 20, 189-196 (2013).

12. Langhorne, P., Coupar, F. \& Pollock, A. Motor recovery after stroke: A systematic review. Lancet Neurol. 8, 741-754 (2009).

13. Lam, P. et al. A haptic-robotic platform for upper-limb reaching stroke therapy: Preliminary design and evaluation results. J. Neuroeng. Rehabil. 5, 1-13 (2008).

14. Urra, O., Casals, A. \& Jane, R. The impact of visual feedback on the motor control of the upper-limb. Proc. Annu. Int. Conf. IEEE Eng. Med. Biol. Society EMBS 2015, 3945-3948 (2015).

15. Hatem, S. M. et al. Rehabilitation of motor function after stroke: A multiple systematic review focused on techniques to stimulate upper extremity recovery. Front. Hum. Neurosci. 10, 1-22 (2016).

16. Thaut, M. H. \& Abiru, M. Rhythmic auditory stimulation in rehabilitation of movement disorders: A review of current research. Music Percept. 27, 263-269 (2010).

17. Spaulding, S. J. et al. Cueing and gait improvement among people with Parkinson's disease: A meta-analysis. Arch. Phys. Med. Rehabil. 94, 562-570 (2013).

18. Ghai, S., Ghai, I. \& Effenberg, A. O. Effect of rhythmic auditory cueing on aging gait: A systematic review and meta-analysis. Aging Dis. 9, 901-923 (2018).

19. Chen, Y. et al. A real-time, multimodal biofeedback system for stroke patient rehabilitation. in Proceedings of the 14th Annual ACM International Conference on Multimedia, MM 2006 501-502 (ACM, 2006). https://doi.org/10.1145/1180639.1180745.

20. Duff, M. et al. An adaptive mixed reality training system for stroke rehabilitation. IEEE Trans. Neural Syst. Rehabil. Eng. 18, 531-541 (2010).

21. McCormick, E. J. \& Sanders, M. S. Human Factors in Engineering and Design (McGraw-Hill, Inc., New York, 1993).

22. Schaefer, R. S. Auditory rhythmic cueing in movement rehabilitation: Findings and possible mechanisms. Philos. Trans. R. Soc. B Biol. Sci. 369, 20130402 (2014).

23. Thaut, M. H. \& Hoemberg, V. Neurologic music therapy in neuropsychological rehabilitation. Neuropsychol. Rehabil. Int. Handb. $2,414-424$ (2017).

24. Spidalieri, G., Busby, L. \& Lamarre, Y. Fast ballistic arm movements triggered by visual, auditory, and somesthetic stimuli in the monkey. II. Effects of unilateral dentate lesion on discharge of precentral cortical neurons and reaction time. J. Neurophysiol. 50, 1359-1379 (1983).

25. Thaut, M. H., Kenyon, G. P., Schauer, M. L. \& McIntosh, G. C. The connection between rhythmicity and brain function. IEEE Eng. Med. Biol. Mag. 18, 101-108 (1999). 
26. Nombela, C., Hughes, L. E., Owen, A. M. \& Grahn, J. A. Into the groove: Can rhythm influence Parkinson's disease?. Neurosci. Biobehav. Rev. 37, 2564-2570 (2013).

27. van Noorden, L. \& Moelants, D. Resonance in the perception of musical pulse. Int. J. Phytoremed. 21, 43-66 (1999).

28. Grahn, J. A. Neural mechanisms of rhythm perception: current findings and future perspectives. Top. Cogn. Sci. 4, 585-606 (2012).

29. Grahn, J. A. See what I hear? Beat perception in auditory and visual rhythms. Exp. Brain Res. 220, 51-61 (2012).

30. Repp, B. H. \& Su, Y. H. Sensorimotor synchronization: A review of recent research (2006-2012). Psychon. Bull. Rev. 20, 403-452 (2013).

31. Fujioka, T., Trainor, L. J., Large, E. W. \& Ross, B. Internalized timing of isochronous sounds is represented in neuromagnetic beta oscillations. J. Neurosci. 32, 1791-1802 (2012).

32. Ross, B., Barat, M. \& Fujioka, T. Sound-making actions lead to immediate plastic changes of neuromagnetic evoked responses and induced $\beta$-band oscillations during perception. J. Neurosci. 37, 5948-5959 (2017).

33. Rochester, L., Baker, K., Nieuwboer, A. \& Burn, D. Targeting dopa-sensitive and dopa-resistant gait dysfunction in Parkinson's disease: Selective responses to internal and external cues. Mov. Disord. 26, 430-435 (2011).

34. Miller, R. A., Thaut, M. H., McIntosh, G. C. \& Rice, R. R. Components of EMG symmetry and variability in parkinsonian and healthy elderly gait. Electroencephalogr. Clin. Neurophysiol. Electromyogr. Mot. Control 101, 1-7 (1996).

35. Besson, M., Chobert, J. \& Marie, C. Transfer of training between music and speech: Common processing, attention, and memory. Front. Psychol. 2, 1-12 (2011).

36. Janata, P., Tillmann, B. \& Bharucha, J. J. Listening to polyphonic music recruits domain-general attention and working memory circuits. Cogn. Affect. Behav. Neurosci. 2, 121-140 (2002).

37. Zatorre, R. J., Chen, J. L. \& Penhune, V. B. When the brain plays music: Auditory-motor interactions in music perception and production. Nat. Rev. Neurosci. 8, 547-558 (2007).

38. Chen, J. L., Zatorre, R. J. \& Penhune, V. B. Interactions between auditory and dorsal premotor cortex during synchronization to musical rhythms. Neuroimage 32, 1771-1781 (2006).

39. Tecchio, F., Salustri, C., Thaut, M. H., Pasqualetti, P. \& Rossini, P. M. Conscious and preconscious adaptation to rhythmic auditory stimuli: A magnetoencephalographic study of human brain responses. Exp. Brain Res. 135, 222-230 (2000).

40. Grahn, J. A. \& Rowe, J. B. Feeling the beat: Premotor and striatal interactions in musicians and nonmusicians during beat perception. J. Neurosci. 29, 7540-7548 (2009).

41. Giovannelli, F. et al. Role of the dorsal premotor cortex in rhythmic auditory-motor entrainment: A perturbational approach by rTMS. Cereb. Cortex 24, 1009-1016 (2014).

42. Hurt, C. P., Rice, R. R., McIntosh, G. C. \& Thaut, M. H. Rhythmic auditory stimulation in gait training for patients with traumatic brain injury. J. Music Ther. 35, 228-241 (1998).

43. Schauer, M. \& Mauritz, K. H. Musical motor feedback (MMF) in walking hemiparetic stroke patients: Randomized trials of gait improvement. Clin. Rehabil. 17, 713-722 (2003).

44. Thaut, M. H., McIntosh, G. C., Prassas, S. G. \& Rice, R. R. Effect of rhythmic auditory cuing on temporal stride parameters and EMG patterns in normal gait. Neurorehabil. Neural Repair 6, 185-190 (1992).

45. Malcolm, M. P., Massie, C. \& Thaut, M. Rhythmic auditory-motor entrainment improves hemiparetic arm kinematics during reaching movements: A pilot study. Top. Stroke Rehabil. 16, 69-79 (2009).

46. Thaut, M. H., Kenyon, G. P., Hurt, C. P., McIntosh, G. C. \& Hoemberg, V. Kinematic optimization of spatiotemporal patterns in paretic arm training with stroke patients. Neuropsychologia 40, 1073-1081 (2002).

47. Wallis, I. et al. Real-time sonification of movement for an immersive stroke rehabilitation environment. in Proceeding of the International Community on Auditory Display 497-503 (2007).

48. Mathias, S. R., Micheyl, C. \& Bailey, P. J. Stimulus uncertainty and insensitivity to pitch-change direction. J. Acoust. Soc. Am. 127, 3026-3037 (2010).

49. Semal, C. \& Demany, L. Individual differences in the sensitivity to pitch direction. J. Acoust. Soc. Am. 120, 3907-3915 (2006).

50. Kang, R. et al. Development and validation of the University of Washington clinical assessment of music perception test. Ear Hear. 30, 411-418 (2009).

51. Tubul, Z. E. N. Musical parameters and children's images of motion. Music. Sci. 14, 89-111 (2010).

52. Larson, S. Musical Forces: Motion, Metaphor, and Meaning in Music (Indiana University Press, Bloomington, 2012).

53. Thaut, M. H. The future of music in therapy and medicine. Ann. N. Y. Acad. Sci. 1060, 303-308 (2005).

54. Thaut, M. H., Trimarchi, P. D. \& Parsons, L. M. Human brain basis of musical rhythm perception: Common and distinct neural substrates for meter, tempo, and patter. Brain Sci. 4, 428-452 (2014).

55. Lamb, J. The Effect of Patterned Sensory Enhancement on Hemiparetic Upper Limb Kinematics (Colorado State University, Fort Collins, 2012).

56. Hamburg, J. \& Clair, A. A. The effects of a movement with music program on measures of balance and gait speed in healthy older adults. J. Music Ther. 40, 212-226 (2003).

57. Hamburg, J. \& Clair, A. A. The effects of a Laban-based movement program with music on measures of balance and gait in older adults. Act. Adapt. Aging 28, 17-33 (2004).

58. Mathews, R. M., Clair, A. A. \& Kosloski, K. Keeping the beat: Use of rhythmic music during exercise activities for the elderly with dementia. Am. J. Alzheimers. Dis. Other Demen. 16, 377-380 (2001).

59. O'Konski, M., Bane, C., Hettinga, J. \& Krull, K. Comparative effectiveness of exercise with patterned sensory enhanced music and background music for long-term care residents. J. Music Ther. 47, 120-136 (2010).

60. Clark, I. N., Baker, F. \& Taylor, N. F. The effects of live patterned sensory enhancement on group exercise participation and mood in older adults in rehabilitation. J. Music Ther. 49, 180-204 (2012).

61. Wang, T. H. et al. A home-based program using patterned sensory enhancement improves resistance exercise effects for children with cerebral palsy: A randomized controlled trial. Neurorehabil. Neural Repair 27, 684-694 (2013).

62. FitzGerald, J. J., Lu, Z., Jareonsettasin, P. \& Antoniades, C. A. Quantifying motor impairment in movement disorders. Front. Neurosci. 12, 202 (2018).

63. Thies, S. B. et al. Movement variability in stroke patients and controls performing two upper limb functional tasks: A new assessment methodology. J. Neuroeng. Rehabil. 6, 2 (2009).

64. De Baets, L., van der Straaten, R., Matheve, T. \& Timmermans, A. Shoulder assessment according to the international classification of functioning by means of inertial sensor technologies: A systematic review. Gait Posture 57, 278-294 (2017).

65. Miller, R. H., Chang, R., Baird, J. L., Van Emmerik, R. E. A. \& Hamill, J. Variability in kinematic coupling assessed by vector coding and continuous relative phase. J. Biomech. 43, 2554-2560 (2010).

66. Haken, H., Kelso, J. A. S. \& Bunz, H. A theoretical model of phase transitions in human hand movements. Biol. Cybern. 51, 347-356 (1985).

67. Kohn, D. \& Eitan, Z. Moving music: Correspondences of musical parameters and movement dimensions in children's motion and verbal responses. Music Percept. 34, 40-55 (2016).

68. Flash, T. \& Hogan, N. The coordination of arm movements: An experimentally confirmed mathematical model. J. Neurosci. $\mathbf{5}$, $1688-1703(1985)$. 
69. Whitall, J., Waller, S. M. C., Silver, K. H. C. \& Macko, R. F. Repetitive bilateral arm training with rhythmic auditory cueing improves motor function in chronic hemiparetic stroke. Stroke 31, 2390-2395 (2000).

70. Luft, A. R. et al. Repetitive bilateral arm training and motor cortex activation in chronic stroke: A randomized controlled trial. J. Am. Med. Assoc. 292, 1853-1861 (2004).

71. Jeong, S. \& Kim, M. T. Effects of a theory-driven music and movement program for stroke survivors in a community setting. Appl. Nurs. Res. 20, 125-131 (2007).

72. Chen, J. L., Fujii, S. \& Schlaug, G. The use of augmented auditory feedback to improve arm reaching in stroke: A case series. Disabil. Rehabil. 38, 1115-1124 (2016).

73. Avanzino, L. et al. Time processing and motor control in movement disorders. Front. Hum. Neurosci. 10, 631 (2016).

74. Schaffert, N., Janzen, T. B., Mattes, K. \& Thaut, M. H. A review on the relationship between sound and movement in sports and rehabilitation. Front. Psychol. 10, 244 (2019).

75. Thaut, M. H., McIntosh, G. C. \& Hoemberg, V. Neurobiological foundations of neurologic music therapy: Rhythmic entrainment and the motor system. Front. Psychol. 5, 1185 (2015).

76. Bella, S. D. et al. Gait improvement via rhythmic stimulation in Parkinson's disease is linked to rhythmic skills. Sci. Rep. 7, 42005 (2017).

77. Braunlich, K. et al. Rhythmic auditory cues shape neural network recruitment in Parkinson's disease during repetitive motor behavior. Eur. J. Neurosci. 49, 849-858 (2019).

78. Hömberg, V. Evidence based medicine in neurological rehabilitation: A critical review. Acta Neurochir. Suppl. 93, 3-14 (2005).

79. Ijmker, T. \& Lamoth, C. J. C. Gait and cognition: The relationship between gait stability and variability with executive function in persons with and without dementia. Gait Posture 35, 126-130 (2012).

80. Wittwer, J. E., Webster, K. E. \& Hill, K. Effect of rhythmic auditory cueing on gait in people with Alzheimer disease. Arch. Phys. Med. Rehabil. 94, 718-724 (2013).

81. Scholz, D. S. et al. Sonification as a possible stroke rehabilitation strategy. Front. Neurosci. 8, 332 (2014).

82. Scholz, D. S. et al. Sonification of arm movements in stroke rehabilitation: A novel approach in neurologic music therapy. Front. Neurol. 7, 106 (2016).

83. Milner, T. E. A model for the generation of movements requiring endpoint precision. Neuroscience 49, 487-496 (1992).

84. Vallbo, A. B. \& Wessberg, J. Organization of motor output in slow finger movements in man. J. Physiol. 469, 673-691 (1993).

85. Krebs, H. I., Aisen, M. L., Volpe, B. T. \& Hogan, N. Quantization of continuous arm movements in humans with brain injury. Proc. Natl. Acad. Sci. 96, 4645-4649 (1999).

86. Rohrer, B. et al. Movement smoothness changes during stroke recovery. J. Neurosci. https://doi.org/10.1523/jneurosci.22-18$08297.2002(2002)$

87. Price, C. I., Rodgers, H., Franklin, P., Curless, R. H. \& Johnson, G. R. Glenohumeral subluxation, scapula resting position, and scapula rotation after stroke: A noninvasive evaluation. Arch. Phys. Med. Rehabil. 82, 955-960 (2001).

88. Meskers, C. G. M., Koppe, P. A., Konijnenbelt, M., Veeger, D. J. E. J. \& Janssen, T. W. J. Kinematic alterations in the ipsilateral shoulder of patients with hemiplegia due to stroke. Am. J. Phys. Med. Rehabil. 84, 97-105 (2005).

89. Niessen, M. H. et al. Proprioception of the shoulder after stroke. Arch. Phys. Med. Rehabil. 89, 333-338 (2008).

90. Niessen, M. et al. Kinematics of the contralateral and ipsilateral shoulder: A possible relationship with post-stroke shoulder pain. J. Rehabil. Med. 40, 482-486 (2008).

91. Kim, J. R. et al. Effects of rhythmic auditory stimulation during hemiplegic arm reaching in individuals with stroke: An exploratory study. Hong Kong J. Occup. Ther. https://doi.org/10.1016/j.hkjot.2014.11.002 (2014).

92. Miniato, M. A. \& Caire, M. J. Anatomy, Shoulder and Upper Limb (Shoulder, StatPearls, 2018).

93. Rundquist, P. J., Dumit, M., Hartley, J., Schultz, K. \& Finley, M. A. Three-dimensional shoulder complex kinematics in individuals with upper extremity impairment from chronic stroke. Disabil. Rehabil. 34, 402-407 (2012).

94. Barnes, C. J., Van Steyn, S. J. \& Fischer, R. A. The effects of age, sex, and shoulder dominance on range of motion of the shoulder. J. Shoulder Elb. Surg. 10, 242-246 (2001).

95. Madgwick, S. O. Automated calibration of an accelerometers, magnetometers and gyroscopes: A feasibility study. Tehc Rep, $\mathrm{x}$-io Technol. Ltd. 1-11, Bristol, UK. http://www.X-io.co.uk/ (report and demonstration video) (2010).

96. Madgwick, S. O. H., Harrison, A. J. L. \& Vaidyanathan, R. Estimation of IMU and MARG orientation using a gradient descent algorithm. in IEEE International Conference on Rehabilitation Robotics 1-7 (2011). https://doi.org/10.1109/ICORR.2011.5975346.

97. Diebel, J. Representing attitude: Euler angles, unit quaternions, and rotation vectors. Matrix 58, 1-35 (2006).

98. Hall, L. C., Middlebrook, E. E. \& Dickerson, C. R. Analysis of the influence of rotator cuff impingements on upper limb kinematics in an elderly population during activities of daily living. Clin. Biomech. 26, 579-584 (2011).

99. Brookham, R. L., Cudlip, A. C. \& Dickerson, C. R. Examining upper limb kinematics and dysfunction of breast cancer survivors in functional dynamic tasks. Clin. Biomech. 55, 86-93 (2018).

100. Chee, R., Murphy, A., Danoudis, M., Georgiou-Karistianis, N. \& Iansek, R. Gait freezing in Parkinson's disease and the stride length sequence effect interaction. Brain 132, 2151-2160 (2009).

101. Gates, D. H., Walters, L. S., Cowley, J., Wilken, J. M. \& Resnik, L. Range of motion requirements for upper-limb activities of daily living. Am. J. Occup. Ther. 70, 7001350010p1-7001350010p10 (2016).

102. Aizawa, J. et al. Ranges of active joint motion for the shoulder, elbow, and wrist in healthy adults. Disabil. Rehabil. 35, 1342-1349 (2013).

103. Rettig, O., Krautwurst, B., Maier, M. W. \& Wolf, S. I. Definition of anatomical zero positions for assessing shoulder pose with 3D motion capture during bilateral abduction of the arms. BMC Musculoskelet. Disord. 16, 383 (2015)

104. Brookham, R. L., Wong, J. M. \& Dickerson, C. R. Upper limb posture and submaximal hand tasks influence shoulder muscle activity. Int. J. Ind. Ergon. 40, 337-344 (2010).

105. Jaspers, E. et al. Upper limb kinematics: Development and reliability of a clinical protocol for children. Gait Posture 33, 279-285 (2011).

106. El-Gohary, M. \& McNames, J. Shoulder and elbow joint angle tracking with inertial sensors. IEEE Trans. Biomed. Eng. 59, 2635-2641 (2012).

107. Wu, C. Y., Chen, C. L., Tang, S. F., Lin, K. C. \& Huang, Y. Y. Kinematic and clinical analyses of upper-extremity movements after constraint-induced movement therapy in patients with stroke: A randomized controlled trial. Arch. Phys. Med. Rehabil. 88, 964-970 (2007).

108. Lin, K. C., Wu, C. Y., Wei, T. H., Lee, C. Y. \& Liu, J. S. Effects of modified constraint-induced movement therapy on reach-tograsp movements and functional performance after chronic stroke: A randomized controlled study. Clin. Rehabil. 21, 1075-1086 (2007).

109. Wu, C. Y., Lin, K. C., Chen, H. C., Chen, I. H. \& Hong, W. H. Effects of modified constraint-induced movement therapy on movement kinematics and daily function in patients with stroke: A kinematic study of motor control mechanisms. Neurorehabil. Neural Repair 21, 460-466 (2007).

110. Kerr, A. \& Rowe, P. An Introduction to Human Movement and Biomechanics (Elsevier, New York, 2019). 


\section{Acknowledgements}

This study was supported by a National Research Foundation of Korea Grant funded by the Korean Government (NRF-2018S1A5A2A03034582).

\section{Author contributions}

E.J. conceived and designed the experiments; J.-H.S. and J.-Y.L. recruited the participants and arranged the individual experiments; S.K. developed the computerised protocol; J.-Y.L. performed the experiments; S.K., E.J., J.L., and I.-Y.K. analysed the data; E.J., S.K., I.-Y.K., J.L., and J.-H.S. interpreted the data; S.K. prepared the tables and figures; E.J., J.-H.S., and S.K. wrote the manuscript; E.J., J.-H.S., S.K., J.L., and I.-Y.K. edited the manuscript; and all authors read and approved the final manuscript.

\section{Competing interests}

The authors declare no competing interests.

\section{Additional information}

Correspondence and requests for materials should be addressed to E.J.

Reprints and permissions information is available at www.nature.com/reprints.

Publisher's note Springer Nature remains neutral with regard to jurisdictional claims in published maps and institutional affiliations.

(c) (i) Open Access This article is licensed under a Creative Commons Attribution 4.0 International License, which permits use, sharing, adaptation, distribution and reproduction in any medium or format, as long as you give appropriate credit to the original author(s) and the source, provide a link to the Creative Commons licence, and indicate if changes were made. The images or other third party material in this article are included in the article's Creative Commons licence, unless indicated otherwise in a credit line to the material. If material is not included in the article's Creative Commons licence and your intended use is not permitted by statutory regulation or exceeds the permitted use, you will need to obtain permission directly from the copyright holder. To view a copy of this licence, visit http://creativecommons.org/licenses/by/4.0/.

(C) The Author(s) 2020 